From Syria to Canada: A Critical Evaluation of Service-Delivery and Coordination along the Journey of Forced Migration

Bernice Tiggelaar ${ }^{1}$, Patricia Strong ${ }^{1}$, Stéphane Michaud ${ }^{1}$, Alison Paul ${ }^{2}$ Salim Sohani ${ }^{1}$, Madeleine Lyons ${ }^{2}$

1. International Operations, Canadian Red Cross, Ottawa/ON/ Canada

2. Disaster Management, Canadian Red Cross, Ottawa/ON/Canada

Study/Objective: The objective of this field research study is to advance learning to improve coordination and service-delivery to crisis-affected populations; highlighted by Canadian Red Cross (CRC) engagement with Syrian populations along the continuum-of-care from Syria to Canada.

Background: Syria is the biggest humanitarian and refugee crisis of our time. Per the United Nations High Commissioner for Refugees (UNHCR), 4.8 million Syrians have fled to surrounding nations, and 6.6 million are internally-displaced. In 2015, the Canadian Prime Minister-elect pledged to bring 25,000 Syrian refugees to Canada. CRC deployed technical personnel along the entire migration journey: Jordan, the Mediterranean Sea, Greece, Germany, and Canada. Service-delivery coordinated by CRC included clinical health, referral, Psychological Support Services (PSS), Restoring-Family-Links (RFL) protection, transportation, lodging and other services.

Methods: End of Mission (EOM) reports $(n=8)$ were analyzed. CRC Syria Response Evaluation was reviewed, which included key informant interviews $(n=24)$, focus groups $(\mathrm{n}=125$ participants), and a survey of volunteers $(\mathrm{n}=583)$. Based on this data and operational experience we have identified recommendations. The EOMs and Response Evaluation will be used to develop an informed set of questions to panel members who can speak to their extensive experience involved in the response; including deployed technical personnel, a Syrian who journeyed from Syria to Canada, and frontline service-providers. Results: Approximately $42 \%$ of the refugees arriving in Canada were assisted by CRC. Challenges included coordination, providing basic-health and PSS during migration, and systemnavigation and referral upon arrival to Canada. Engaging at various points along the migration journey provided unique opportunities for RFL. Recommendations arising from both successes and challenges included: ensuring human-resource systems are prepared; increasing focus on managing health, including child PSS; and using international experience to improve reintegration services.

Conclusion: Knowledge generated from this response models challenges and solutions in supporting service-delivery and coordination with populations affected by crisis throughout the migration journey.

Prehosp Disaster Med 2017;32(Suppl. 1):s77-s78

doi:10.1017/S1049023X17002059

ANA Light Field Hospital: A New Model of Civilian Cooperation and Response during Disasters, Emergencies in Austere Environments, Italy

Alessandra Rossodivita ${ }^{1}$, Federica De Giuli ${ }^{2}$, Antonio Tonarelli ${ }^{2}$, Giuliano Rizzardini ${ }^{1}$, Sergio Rizzini ${ }^{2}$
1. Infectious Diseases Dpt., Luigi Sacco University Hospital, Milan/ Italy

2. Presidio Matteo Rota, GIMC -Medical and Surgical Intervention Group - Ospedale da Campo A.N.A. Italian Association of Alpini, Bergamo/Italy

Study/Objective: The authors describe an Italian Model of Mobile Light Field Hospital of ANA (The Italian Association of Alpini) as a flexible, mobile hospital structure, self contained and self sufficient health care design for rapid deployment, expansion or contraction. This structure is designed to work, and to be deployed in disasters/emergencies. The Field Light Hospital is a new conceptual hospital, designed in a new architectural structure, lighter and easy to use to support of the local emergency services. Civilian personnel are trained as volunteers to work together, improving training programs in emergency preparedness and response.

Background: During disasters/emergencies local health services can be overwhelmed, and damage to clinics and hospitals can render them extremely unsecure or useless. Lessons from past complex disasters such as civil conflicts, wars, and humanitarian emergencies show that Field Hospitals(FHs) as temporary hospitals, civilian or military, plays a significant response role in disasters. Methods: The Hospital on the Field of Alpini actually operates in Italy in support to the activities of civil protection, but also works in different national and international context, based on the activity of volunteers, experts in maxi-emergency, critical medicine and with military tactical backgrounds.

Results: The Alpini Light Field hospital shows a new model of civilian cooperation, and is able to be rapidly deployed in national and international (long) missions. The authors would like to underline how, during complex disasters the need of a rapid public health response, is crucial to prevent the occurrence of new cases, coupled with treatment of victims and maintenance of a viable healthcare delivery system.

Conclusion: During disasters it is useful to utilize FH as support to population affected countries, suggesting a new form of civilian cooperation in support of civil protection activity; and also implementing a new form of research program in training, preparedness and response.

Prehosp Disaster Med 2017;32(Suppl. 1):s78

doi:10.1017/S1049023X17002060

\section{A Multi-Level and Multi-Sectoral Coordination for an Effective Response to the Cholera Outbreak in Central African Republic \\ Michel N.K. Yao}

Who Country Office In Car, World Health Organization, Bangui/ Central African Republic

Study/Objective: This paper aims at sharing a lesson from a specific coordination mechanism to control a cholera outbreak that could have been a major one, due to all the risk factors and the weak response capacity.

Background: A cholera outbreak that started on July 27, 2016 (vibrio Cholerae, serotype O1 Inaba), was declared on August 10, 2016, in the southern part of Central African Republic 
(CAR), in a context of a weak, safe water supply system (less than $55 \%$ people in rural areas in 2015), and a poor sanitation system (7\% people in rural areas), as well as a weak capacity to deliver health services. The outbreak reached the capital city exposing 1.7 million people and 243,000 Internally Displaced Persons (IDPs). A strong multi-sectoral coordination was set up.

Methods: A case study on a field experience.

Results: Twenty deaths and 265 persons were notified as the result of the cholera outbreak from week 27 to 37 . Three levels of coordination:

The Public Health Emergency Operation Center (COUSP) where a technical strategic coordination was taking place to define and implement the response plan. Within the COUSP different experts, decision makers and support teams were analyzing the situation and organizing means for the response, including the rapid response teams, community engagement and communication to stop the spread.

Taskforce cholera is made up of different humanitarian relevant cluster partners (Health, WASH, Camp management, Food Security/nutrition, education and logistic), and implementing technical advices from the COUSP including case investigation and management.

The Outbreak multi-sectoral committee that involves national relevant ministerial departments to ensure joint interventions.

Conclusion: A strong technical and operational coordination contributed to mobilizing all available resources, and guide the response in order to win the race against cholera and avoid further risk to about 1.7 million. This experience should guide future responses to disease outbreaks.

Prehosp Disaster Med 2017;32(Suppl. 1):s78-s79

doi:10.1017/S1049023X17002072

Developing Smart Practices for Prehospital Field

Staff in Situations of Insecurity through Knowledge

Co-Construction

Panu Saaristo ${ }^{1}$, Reda Sadki ${ }^{2}$, Frederik Siem ${ }^{3}$

1. Health And Care, International Federation of Red Cross and Red Crescent Societies, Geneva/Switzerland

2. Geneva Learning Foundation, Geneva/Switzerland

3. Norwegian Red Cross, Oslo/Norway

Study/Objective: The Community of Action for Ambulance and Prehospital Emergency Care Providers in Risk Situations is a network of experts and other relevant actors, committed to improving the operational security in situations of insecurity, in order to better access people in need of emergency medical assistance. There is a lack of high-quality case studies that document the experience and practice of prehospital care.The Community of Action requires effective tools to build its network, develop highquality knowledge, and foster learning between its members.

Background: Almost irrespective of the country or the services, prehospital care providers risk being exposed to violence and/or the threat of violence. The extent and frequency of the violence will vary, but threats, insults and physical attacks are a reality for ambulance and prehospital personnel even in the most peaceful contexts. Considering the serious consequences of such violence, there has been very little research done on this problem, and the solutions that have been developed to address it. Therefore, it is challenging to advocate for change and help the providers to safely deliver on-the-job that they are mandated to do.

Methods: A 4-week digital course using Scholar - a system for learning through knowledge co-construction, was developed in partnership with the Geneva Learning Foundation.

Results: Each participant developed a draft case study, then peer reviewed the drafts of three of their colleagues, and finally revised their initial draft using the feedback from their peers. Through the four weeks, participants engaged in a private, shared space for dialogue.

Conclusion: The Scholar process, based on peer review that models how prehospital practitioners solve problems, learn and collaborate, produces an immediate benefit for those involved as both authors and reviewers; quality; strengthened professional relationships that outlast the process and productive diversity.

Prehosp Disaster Med 2017;32(Suppl. 1):s79

doi:10.1017/S1049023X17002084

Teaching Public Health in Disasters using Massive Open Online Course and Building the Global Humanitarian Response Community

Chunlan Guo, Emily Y.y. Chan, Zhe Huang, Gloria K.w. Chan, Hale H.l. Ho

Collaborating Centre For Oxford University And Cuhk For Disaster And Medical Humanitarian Response, The Chinese University of Hong Kong, Hong Kong/Hong Kong Prc

Study/Objective: To reveal the spatial and temporal pattern of student enrollment in the Public Health Principles in Disaster and Medical Humanitarian Response (PHPID), Massive Open Online Course (MOOC), and to examine the degree to which socio-demographic variables can predict the course achievement.

Background: The first cohort of the PHPID online course was launched by Collaborating Centre for Oxford University and CUHK for Disaster and Medical Humanitarian Response in June 2014. This online course aims to enable students to gain insight and theoretical understanding of the public health issues related to disaster and medical humanitarian relief in the Asia Pacific region, through making lessons learned from previous disasters.

Methods: This study collected registration data from the four completed cohorts during June 2014 to May 2016. The registration data consists of participants' socio-demographic factors, residential location, related experience in disaster response, reason of taking, and the channel of 'first hear' the course. Descriptive and multiple logistic regressions were conducted via SPSS.

Results: In total, 3,457 participants, from $150+$ different countries, registered in the PHPID Model platform; 711 completed and obtained certificates, and 510 left contact information for further collaboration. The most 10 frequently reported countries of origin were consistent with natural disaster hotspots. The first month of each cohort was a peak period of new registrations. Generally, men were $27 \%$ more intent to complete the course $(\mathrm{OR}=1.268,95 \% \mathrm{CI}$ : 1.068 1.505). Moreover, the participants, who have achieved the 